Deconstructing Oligomer Distributions: Discrete Species and Artificial Distributions Peer-reviewed author version

DE NEVE, Jeroen; HAVEN, Joris; Harrisson, Simon \& JUNKERS, Tanja (2019) Deconstructing Oligomer Distributions: Discrete Species and Artificial Distributions. In: ANGEWANDTE CHEMIE-INTERNATIONAL EDITION, 58, p. 1-6.

DOI: 10.1002/anie.201906842

Handle: http://hdl.handle.net/1942/29090 


\title{
Deconstructing oligomer distributions: discrete species and artificial distributions
}

\author{
Jeroen De Neve, Joris J. Haven, Simon Harrisson, Tanja Junkers*
}

\begin{abstract}
The separation of an oligo(methyl acrylate) distribution - obtained from reversible addition-fragmentation chain transfer (RAFT) polymerization - in a discrete (dispersity $=1$ ) oligomeric library (degree of polymerization between 1 and 22) is presented. The properties of this library, in terms of diffusivity, glass transition temperature and viscosity, are determined, filling a significant knowledge gap associated with these materials. Further, the obtained oligomer library is used to construct artificial oligomer distributions on demand. These artificial oligomer distributions are used to highlight the potential to tailor physical properties of a material, while concomitantly demonstrating the limitations associated with size exclusion chromatography analysis of molecular weight and dispersity in particular.
\end{abstract}

The last decade has been marked by the rapid development of precision polymer synthesis. Discrete oligomers are regarded as the "Holy Grail" in polymer science, due to their enormous potential as catalysts (artificial enzymes), as molecular recognition agents (artificial lectins) and as molecular stores of information (artificial DNA). ${ }^{[1-2]}$ As a consequence various synthesis strategies - relying on iterative and orthogonal growth, step-growth or reversible deactivation radical polymerization (RDRP) - have been developed to date and applications in drug delivery and catalysis are currently being explored. ${ }^{[3-5]}$ Nevertheless, no systematic data is available yet on the properties of discrete synthetic oligomers to allow for a better fundamental understanding of their physical and thermal characteristics, such as diffusivity, glass transition temperature, viscosity, crystallinity and rheological behavior. Here, a number of these properties are studied.

Furthermore, mixing discrete oligomers results in artificial distributions, which can be used to tune material properties in a non-chemical pathway. The oligomers are obtained via the deconstruction of a conventional polymer distribution. These components can then be recombined into any desired distribution.

[a] J. De Neve, Dr. J. J. Haven, Prof. T. Junkers

Polymer Reaction Design Group, School of Chemistry Monash University

19 Rainforest Walk, Building 23, Clayton, VIC 3800 (Australia) E-mail: tanja.junkers@monash.edu

[b] J. De Neve, Prof. T. Junkers

Institute for Materials Research

Hasselt University

Martelarenlaan 42, 3500 Hasselt (Belgium)

[c] Dr. S. Harrisson

Laboratoire des IMRCP CNRS UMR 5623

University of Toulouse

118 Route de Narbonne

31062 Toulouse Cedex 9 (France)

Supporting information and the ORCID identification number(s) for the author(s) can be found under:
RDRP is typically fast and doesn't require protection/deprotection chemistry. The disperse mixtures obtained are separated into discrete oligomers via flash column chromatography, a strategy introduced by Hawker and coworkers. ${ }^{[6-8]}$ This allows to study thermal and physical characteristics of oligomers with a stable hydrocarbon backbone that are chemically identical to well-known disperse synthetic polymers. ${ }^{[8]}$

The properties of disperse acrylic polymers have been widely investigated. ${ }^{[9-11]}$ For high-performance applications - in fields such as data storage, ${ }^{[12]}$ catalysis $^{[13]}$ or pharmacology $[5,14]$ - stringent prerequisites necessitate absolute control over material characteristics together with the capability to tune material properties. ${ }^{[15]}$ Parameters such as diffusion coefficients, intrinsic viscosities and glass transition temperatures are of high interest for discrete species, since to date only average values are known, hampering the theoretical understanding of chain-length dependencies. Being able to provide a full set of physical data for a series of oligomers will enable a better understanding of scaling laws and fundamentals of polymer physics.

RAFT was used to prepare a discrete oligo(methyl acrylate) library ranging from 1 to 22 methyl acrylate monomer insertions (degree of polymerization DP $=1-22$ ). These unimers (1mer) to docosamers (22mer) are from here on referred to as DP 1 to 22 . The discrete oligo(methyl acrylate)s obtained are expected to behave substantially differently from their disperse high molecular weight polymeric counterparts, due to being monodisperse, but mostly also due to not having reached the length yet that is required to form ideal polymer coils. The transition between oligomers and polymers is not precisely defined, but the general consensus is that it is located around 50 to 100 monomer units, when chains switch from a pure Rouse motion towards more reptation-like behaviour according to Edwards' tube model in polymer melts. ${ }^{[16]}$ This transition towards oligomers coincides with a much stronger dependency of physical properties on molecular weight. ${ }^{[17]}$ With discrete oligomers at hand, it is also possible to create precisely defined artificial oligomer distributions. The design of tailored molecular weight distributions (MWD) has found substantial interest over the years as the MWD governs the resulting thermal and mechanical properties. ${ }^{[18-19]}$ Further, artificial distributions can be potentially used to encode information in a simple fashion in a bar-code like manner. Various examples in literature exist to tailor unusual MWDs, yet they all start from an inherently disperse polymer mixture. ${ }^{[20-21]}$

The use of discrete oligomers also provides insight into MWD analysis via size exclusion chromatography (SEC). Polymer distributions are typically characterized by their 


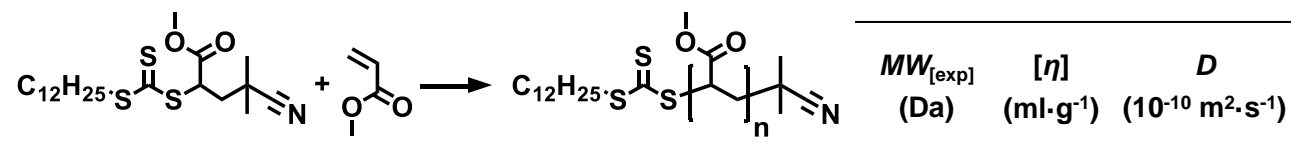
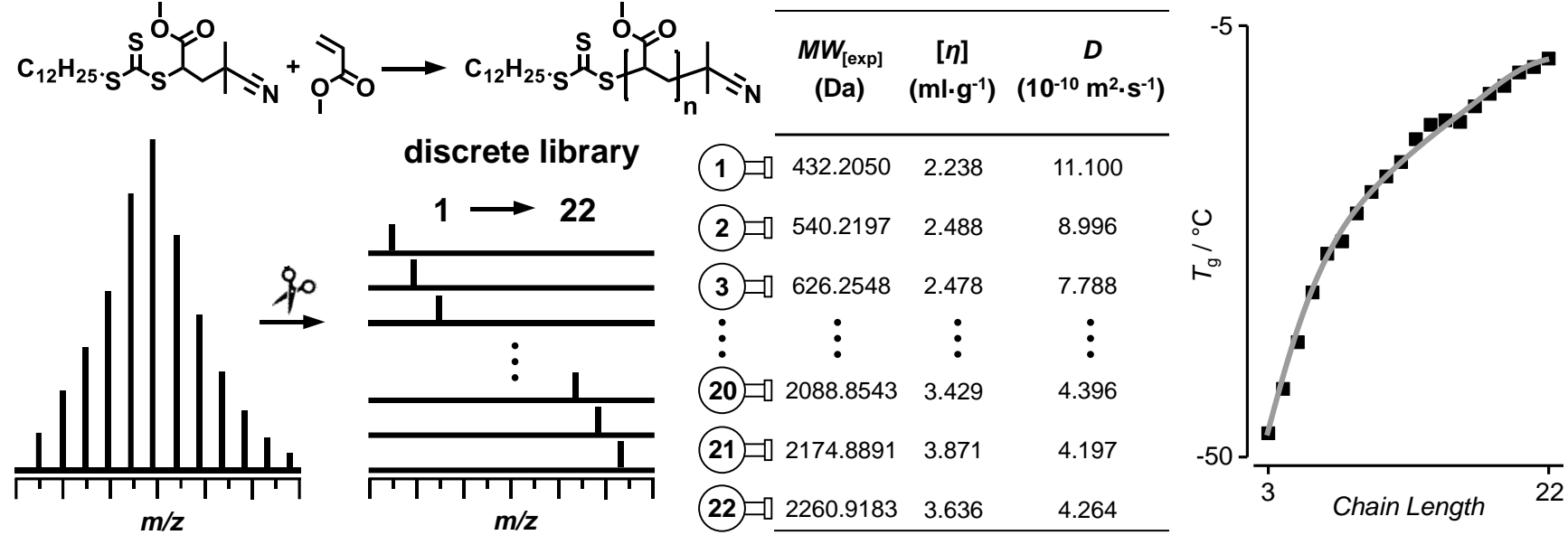

Figure 1. Development of a discrete methyl acrylate oligomer library with a total of 22 entries via reversible addition-fragmentation chain-transfer polymerization followed by flash column chromatography purification. All oligomers were characterized by nuclear magnetic resonance, size-exclusion chromatography, differential scanning calorimetry and mass spectrometry to report on physical parameters such as intrinsic viscosity, diffusion coefficient and glass transition temperature in function of chain length. $M W_{\text {[exp] }}=$ experimentally determined molecular weight via mass spectrometry, $[\eta]=$ intrinsic viscosity, $D=$ diffusion coefficient, $T_{g}=$ glass transition temperature.

number-average molecular weight, $M_{\mathrm{n}}$, and dispersity, $\oplus$. For narrow distributions, $(\Theta<1.5)$, the standard deviation, $\sigma$, provides a more intuitive measure of dispersion than $\oplus^{[22]}$ In order to generate the oligomer library a crude oligomer mixture obtained from RAFT polymerization of methyl acrylate (MA) (see the Supporting Information for experimental details) was used as starting point. Subsequently, flash column chromatography, as first introduced by Hawker as oligomer separation method, ${ }^{[5]}$ was employed to isolate discrete oligomers in the molecular weight range of $432(\mathrm{DP}=1)$ to $2261 \mathrm{~g} \cdot \mathrm{mol}^{-1}(\mathrm{DP}=22)$ (Figure 1). With increasing DP the relative difference in polarity progressively diminishes, which limits the maximum length of oligomers that can be purified. The library prepared here includes an oligomer of DP 22 (docosamer) which is to the best of our knowledge - by far the longest isolated oligomer reported via RDRP. DP 22 corresponds to a length often used in block copolymers. ${ }^{[23-24]}$

The influence of oligomer chain length on thermal properties was studied via differential scanning calorimetry (DSC) as shown in Figure 1 (see the SI for experimental details). A glass transition $\left(T_{\mathrm{g}}\right)$ was apparent from DP $3\left(-47.5^{\circ} \mathrm{C}\right)$ to $22\left(-8.4{ }^{\circ} \mathrm{C}\right)$ as can be expected for non-crystalline oligo(methyl acrylate) (PMA). ${ }^{[25]}$ While literature values for the $T_{\mathrm{g}}$ of PMA - with $M_{\mathrm{n}}$ of $76500 \mathrm{~g} \cdot \mathrm{mol}^{-1}$ and $\Theta$ of $1.26-$ are situated around $10^{\circ} \mathrm{C},{ }^{[26]}$ much lower $T_{\mathrm{g}}$ were measured for the small DP oligomers. ${ }^{[7]}$ This trend is in good agreement with the Flory-Fox equation that is applicable to higher molecular weight polymers. ${ }^{[27]}$

$$
T_{\mathrm{g}}=T_{\mathrm{g}, \infty}-\frac{\mathrm{K}}{M_{\mathrm{n}}}
$$

With $T_{\mathrm{g}, \infty}$ the limiting $T_{\mathrm{g}}$ at infinite molar mass and $\mathrm{K}$ the Flory-Fox constant (related to the free volume of the polymer matrix $\left.{ }^{[28]}\right)$, which are $(5.6 \pm 0.5)^{\circ} \mathrm{C}$ and $(3.39 \pm 0.07) \times 10^{4}{ }^{\circ} \mathrm{C}$ $\mathrm{g} \cdot \mathrm{mol}^{-1}$ respectively for the discrete oligomers. The FloryFox constant is consistent with literature values. ${ }^{[29-30]}$ Thus, shorter oligomer chains with a higher abundance of chain end units contribute to an increased free volume and consequently lower $T_{\mathrm{g}}$. The $T_{\mathrm{g}}$ for the crude oligomer mixture before fractionation was $-14.2^{\circ} \mathrm{C}$.
Knowledge on intrinsic viscosities is of high importance for the molecular weight analysis of polymers. For high molecular weight polymers (> 10000 g.mol-1) molecular weight and intrinsic viscosity are related by the MarkHouwink-Kuhn-Sakurada (MHKS) equation.

$$
[\eta]=\mathrm{K} \times \mathrm{M}^{\alpha}
$$

with $[\eta]$ the intrinsic viscosity, $M$ the molecular weight and the Mark-Houwink parameters $\mathrm{K}$ and $\alpha$. While the MHKS equation is generally only valid for polymers of substantial length, an increase in intrinsic viscosity was still observed for our longer oligomers (see the Supporting Information). The resulting data obtained in THF at $40^{\circ} \mathrm{C}$ fitted the MHKS equation with $\alpha$ of $(0.30 \pm 0.02)$ and $K$ of $(0.37 \pm 0.04) \mathrm{mL} \mathrm{g}$ 1 . This is a considerable deviation from literature values for $\alpha$ of poly(methyl acrylate) under otherwise identical conditions. ${ }^{[31]}$ The lower value of $\alpha$ indicates that oligomers adopt a more compact conformation than their polymeric counterparts in THF solution. Due to their insufficient length, the oligomers adopt worm-like configurations rather than forming statistical polymer coils. Interestingly, for the intercept of the MHKS fit with literature values for higher MW polymers, one can calculate the cross-over chain length (see SI, Figure S28), from where on oligomers become

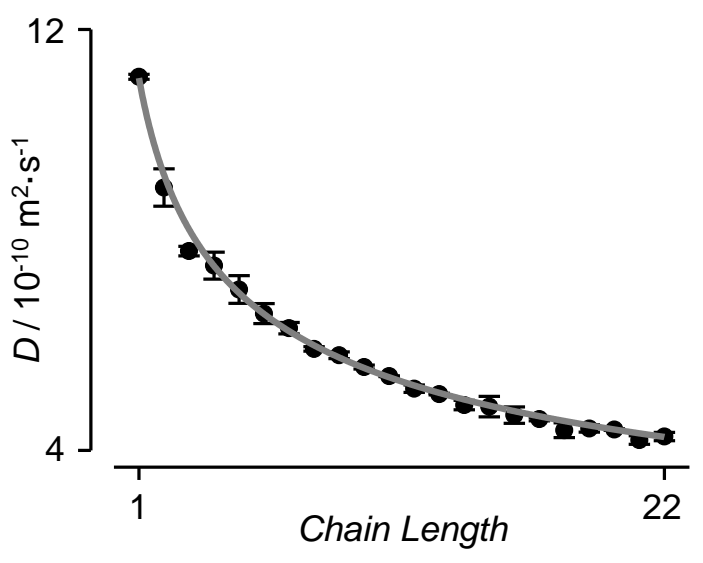

Figure 2. Diffusion coefficients as obtained for every individual oligomer chain length through diffusion ordered spectroscopy. 
polymers, in the sense of forming random coils. This length is determined to be around 37 chain units. While speculations had been made that the transition should occur in this range ${ }^{[32]}$ no reliable method of determination had been found so far.

Figure 2 shows diffusion coefficients obtained through diffusion ordered NMR spectroscopy (DOSY) measurements in deuterated chloroform. Diffusion coefficients provide a measure of the mobility of the oligomer chains. Knowledge of diffusivity for oligomeric species would remove the need for several assumptions that are typically made in e.g. the theory of bimolecular termination rate coefficients in radical polymerizations. The highest self-diffusion of $11.1 \times 10^{-10} \mathrm{~m}^{2} \cdot \mathrm{s}^{-1}$ is measured for DP 1 after which a logarithmic decrease is observed with a final value of $4.3 \times 10^{-10} \mathrm{~m}^{2} \cdot \mathrm{s}^{-1}$ for DP 22 (Figure 2). The diffusion coefficient of the starting oligomer mixture before fractionation was measured to be $4.8 \times 10^{-10} \mathrm{~m}^{2} \cdot \mathrm{s}^{-1}$. These diffusion coefficients are substantially higher than those of high molecular weight polydisperse samples, demonstrating the expected increased mobility of oligomeric chains. ${ }^{\left[{ }^{32]}\right.}$ The best fit of the data to $D=k(\mathrm{DP}+x)^{-a}$ (where $\mathrm{x}$ represents a fitted parameter to account for the presence of the RAFT group) yields an exponent of $-0.36 \pm 0.01$ (Figure 2), which is in remarkably good agreement with literature data on the termination rate coefficient in methyl acrylate polymerization in this chain length regime (note that the chain length dependency of termination and diffusion is directly correlated). ${ }^{[33]}$ Again, this provides for the first time a direct and unambiguous determination of data that had to date only been derived in very indirect manner and that had remained subject to speculation. It should be noted that all DOSY experiments were performed in deuterated chloroform while SEC experiments were conducted in THF due to practical considerations.

Mechanical properties such as plasticity and elasticity are directly dependent on the MWD. Over the past couple of years, the topic of systematically varying dispersity and - to a lesser extent - purposefully manipulating MWDs obtained via RDRP has caught the attention of the scientific community. ${ }^{[34]}$ However explicit knowledge of the relative contribution of each chain length to the overall distribution could not be obtained due to constraints in the availability of proper starting materials and adequateness of synthetic methods applied. Studying artificial polymer distributions arising from a deconstructed oligomer library not only allows a deeper understanding of molecular weight analysis performed via SEC, it also acts as an example of how material properties could be tuned to achieve desired characteristics at will.

With this method experimentally obtained molecular weight distributions can be deconstructed, and subsequently reconstructed in any shape. To showcase the versatility of this strategy unusual distributions were produced that would normally be inaccessible by any controlled polymerization technique. As a first example we mixed $0.25 \mu \mathrm{mol} \cdot \mathrm{ml}^{-1}$ of each oligomer with DP 1 to 5 and 18 to 22 together with 0.10 $\mu \mathrm{mol} \cdot \mathrm{ml}^{-1}$ of oligomers with DP 6 to 17 as shown in Figure 3 (see the $\mathrm{SI}$ for more examples). This gives a theoretical number distribution containing 2 peaks at either end of the molecular weight range connected by a lower bridge formed by chains of average length (Figure $3 a$ ). In the resulting number distribution after SEC analysis (Figure $3 b$ ) $M_{n}$ calculated from the moments of the SEC distribution displayed very good agreement with the theoretically expected value. Yet, the overall shape of the MWD is quite different from what some might expect on first glance. The reason for the discrepancy between experimental number distributions and the envisioned distribution is rooted in a band broadening effect that mainly stems from the purely physical effect of axial dispersion. In addition a difference in elution for diastereoisomers could have a minor influence which is currently being studied in our research labs. This leads to the result that even discrete oligomers appear as distributions in SEC (with $Ð$ around 1.02) and hence single oligomer profiles overlap. Due to the logarithmic nature of SEC separation, overlaps elevate higher MW areas more than lower MW areas. With the input of all oligomer profiles
A
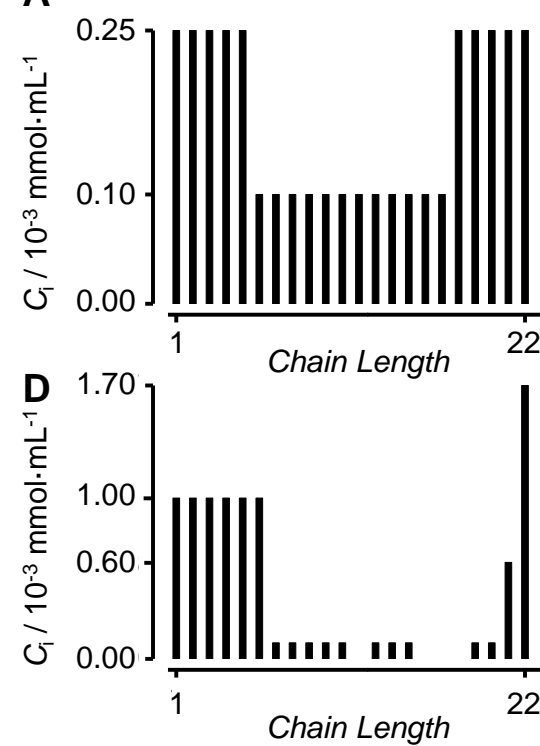

B

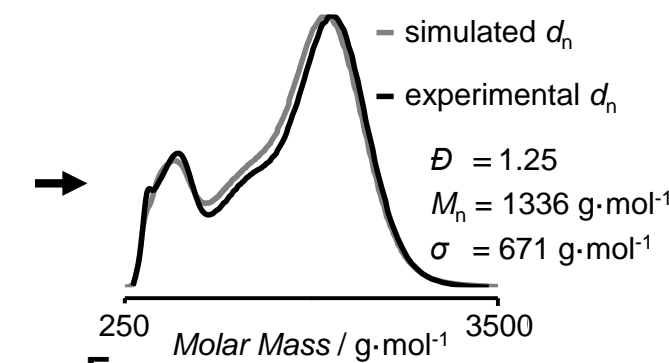

C

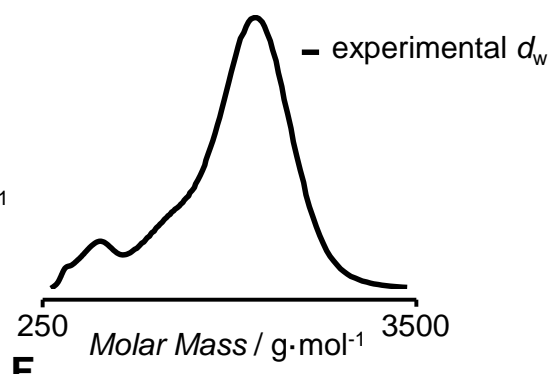

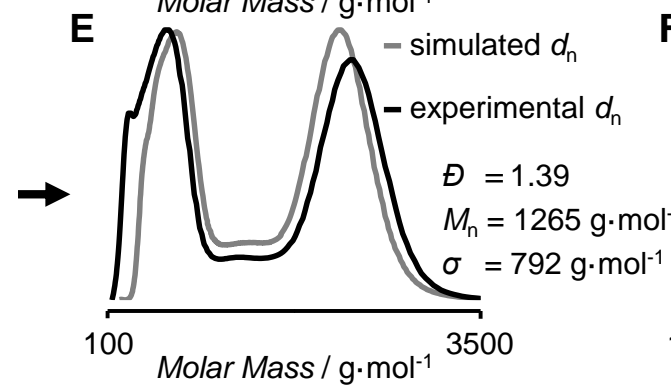

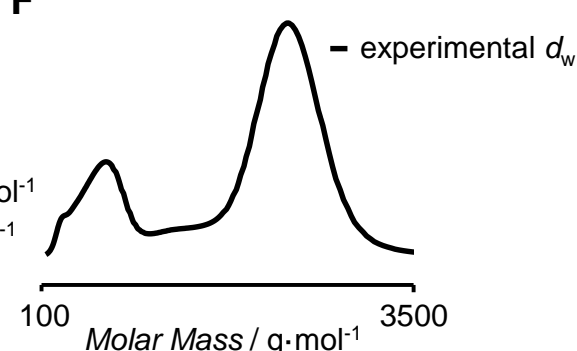

Figure 3. Schematic representation of the recombination of different chain lengths. A, D) Specific ratios of each discrete oligomer were mixed to create designer distributions. B, E) The resulting number distributions are displayed with a comparison between the simulated result and the experimentally obtained data. C, F) The resulting double weighted SEC distributions. $C_{i}=$ concentration, $d_{n}=$ number distribution, $d_{w}=$ double weighted SEC distribution, $D=$ dispersity, $M_{n}=$ number average molecular weight, $\sigma=$ standard deviation. 
obtained from the MHKS plot measurements, an improved simulation of full MWDs is obtained which is nearly identical to the experimental observations (Figure $3 \mathrm{~b}$ and $3 \mathrm{e}$ ). This highlights that even if the components are low in apparent dispersity, band broadening and peak overlaps need to be accounted for to accurately predict overall MWDs. As can be seen in Figure $3 d$ and $3 e$, a significantly different theoretical number distribution is required to reach the desired SEC-measured number distribution. For this oligomer mixture a $T_{\mathrm{g}}$ of $-43.6^{\circ} \mathrm{C}$ was determined, which is significantly lower than the expected $T_{\mathrm{g}}$ of $-21.3{ }^{\circ} \mathrm{C}$, calculated from the $M_{\mathrm{n}}$ and the Flory-Fox equation. This further demonstrates that not only the average molecular weight must be taken into account, but also the shape and broadness of the MWD. This is in good agreement with a previous study where we had shown that $T_{\mathrm{g}}$ is dependent on dispersity. ${ }^{[7]}$

It is remarkable that even though exotic distributions with very substantial multimodality are created (see SI for further examples), in most cases the overall dispersities remain in the expected range for a well-controlled polymerization (e.g. $<1.3$ ), and all are $<1.5$. This is in agreement with previous reports that dispersity is a misleading measure of the homogeneity of polymer samples, especially in the short chain length regime. Standard deviation has been proposed as a more intuitive indicator.[22] For the example given in Figure $3 b$, the standard deviation of the distribution is 671 $\mathrm{g} \cdot \mathrm{mol}-1$ for an $\mathrm{Mn}$ of $1336 \mathrm{~g} \cdot \mathrm{mol}-1(\Xi=1.25)$. From $\sigma$, it is immediately evident that a highly non-uniform mixture has been obtained, while the dispersity alone gives a counterintuitive impression. In fact, we were unable to create "unacceptably" high dispersities - defined as $Đ>1.5$ - using our library. Understanding this deceptive feature of MWD dispersity is highly important as more and more research is dedicated to the creation of precision polymers carrying specific compositions and defined spatial arrangements of functionalities.

\section{Acknowledgements}

The authors are grateful for helpful discussions with Peter Nichols regarding DOSY NMR and with Maarten Rubens for the simulation of SEC number distributions. They would also like to kindly acknowledge Kei Saito for granting access to their DSC instrument. The authors thank Andres Castro Villavicencio for his help with preliminary experiments. J.D.N. is grateful to the Bijzonder Onderzoeks Fonds (BOF) of Hasselt University for providing a scholarship. Funding from Monash University is also kindly acknowledged.

Keywords: discrete polymers $\cdot$ RAFT polymerization • dispersity $\bullet$ diffusion $\bullet$ glass transition J.-F. Lutz, M. Ouchi, D. R. Liu, M. Sawamoto, Science 2013, 341,1238149

J.-F. Lutz, J.-M. Lehn, E. W. Meijer, K. Matyjaszewski, Nature Reviews Materials 2016, 1, 16024

[3] J. De Neve, J. J. Haven, L. Maes, T. Junkers, Polymer Chemistry 2018, 9, 4692-4705.

[4] S. C. Solleder, R. V. Schneider, K. S. Wetzel, A. C. Boukis, M. A. R. Meier, Macromolecular Rapid Communications 2017, 38, 1600711 .

[5] E. Maron, J. H. Swisher, J. Haven, T. Y. Meyer, T. Junkers, H. G. Börner, Angewandte Chemie International Edition, 0. J. J. Haven, J. A. De Neve, T. Junkers, ACS Macro Letters

2017, 6, 743-747.
J] J. Haven, T. Junkers, Polymer Chemistry 2019, 10, 679682
[8] J. Lawrence, S.-H. Lee, A. Abdilla, M. D. Nothling, J. M. Ren, A. S. Knight, C. Fleischmann, Y. Li, A. S. Abrams, B. V. K. J. Schmidt, M. C. Hawker, L. A. Connal, A. J. McGrath, P. G. Clark, W. R. Gutekunst, C. J. Hawker, Journal of the American Chemical Society 2016, 138, 6306-6310.

[9] J. M. Ren, J. Lawrence, A. S. Knight, A. Abdilla, R. B. Zerdan, A. E. Levi, B. Oschmann, W. R. Gutekunst, S.-H. Lee, Y. Li, A. J. McGrath, C. M. Bates, G. G. Qiao, C. J. Hawker, Journal of the American Chemical Society 2018, 140, 1945-1951.

[10] E. A. Campo, in Selection of Polymeric Materials (Ed.: E. A. Campo), William Andrew Publishing, Norwich, NY, 2008, pp. 1-39.

[11] J. Lawrence, E. Goto, J. M. Ren, B. McDearmon, D. S. Kim, Y. Ochiai, P. G. Clark, D. Laitar, T. Higashihara, C. J. Hawker, Journal of the American Chemical Society 2017, 139, 1373513739 .

[12] S. Martens, A. Landuyt, P. Espeel, B. Devreese, P. Dawyndt, F. Du Prez, Nature Communications 2018, 9, 4451.

[13] J. W. Kramer, D. S. Treitler, E. W. Dunn, P. M. Castro, T.

Roisnel, C. M. Thomas, G. W. Coates, Journal of the American Chemical Society 2009, 131, 16042-16044.

[14] P. R. Judzewitsch, T.-K. Nguyen, S. Shanmugam, E. H. H. Wong, C. Boyer, Angewandte Chemie International Edition 2018, 57, 4559-4564.

[15] G. Moriceau, J. Tanaka, D. Lester, G. S. Pappas, A. B. Cook P. O'Hora, J. Winn, T. Smith, S. Perrier, Macromolecules 2019.

[16] M. E. Cates, Macromolecules 1987, 20, 2289-2296.

[17] G. S. Grest, The Journal of Chemical Physics 2016, 145, 141101

[18] N. Corrigan, A. Almasri, W. Taillades, J. Xu, C. Boyer, Macromolecules 2017, 50, 8438-8448.

[19] D. T. Gentekos, J. Jia, E. S. Tirado, K. P. Barteau, D.-M. Smilgies, R. A. DiStasio, B. P. Fors, Journal of the American Chemical Society 2018, 140, 4639-4648.

[20] N. Corrigan, R. Manahan, Z. T. Lew, J. Yeow, J. Xu, C. Boyer, Macromolecules 2018, 51, 4553-4563.

[21] D. T. Gentekos, L. N. Dupuis, B. P. Fors, Journal of the American Chemical Society 2016, 138, 1848-1851.

[22] S. Harrisson, Polymer Chemistry 2018, 9, 1366-1370.

[23] T. Chang, M. S. Lord, B. Bergmann, A. Macmillan, M. H. Stenzel, Journal of Materials Chemistry B 2014, 2, 28832891.

[24] C. Zhang, Y. Zhu, C. Zhou, W. Yuan, J. Du, Polymer Chemistry 2013, 4, 255-259.

[25] J. Hintermeyer, A. Herrmann, R. Kahlau, C. Goiceanu, E. A. Rössler, Macromolecules 2008, 41, 9335-9344.

[26] K. B. Guice, Y.-L. Loo, Macromolecules 2007, 40, 9053-9058.

[27] T. G. F. Jr., P. J. Flory, Journal of Applied Physics 1950, 21 , 581-591.

[28] V. N. Novikov, E. A. Rössler, Polymer 2013, 54, 6987-6991.

[29] R. F. Boyer, Macromolecules 1974, 7, 142-143.

[30] B. Metin, F. D. Blum, The Journal of Chemical Physics 2006, $124,054908$.

[31] T. Gruendling, T. Junkers, M. Guilhaus, C. Barner-Kowollik, Macromolecular Chemistry and Physics 2010, 211, 520-528.

[32] N. Cherifi, A. Khoukh, A. Benaboura, L. Billon, Polymer Chemistry 2016, 7, 5249-5257.

[33] A. Theis, A. Feldermann, N. Charton, M. H. Stenzel, T. P. Davis, C. Barner-Kowollik, Macromolecules 2005, 38, 25952605.

[34] V. Yadav, N. Hashmi, W. Ding, T.-H. Li, M. K. Mahanthappa, J. C. Conrad, M. L. Robertson, Polymer Chemistry 2018, 9 4332-4342. 